\title{
Remote Activity Monitoring Using Indirect Sensing Approach in Assisted Living Scenario
}

\author{
Heikki Karvonen \\ University of Oulu \\ Centre for Wireless Communications \\ Oulu, Finland \\ heikki.karvonen@oulu.fi
}

\author{
Arto Matilainen \\ University of Oulu \\ Centre for Wireless Communications \\ Oulu, Finland \\ arto.matilainen@oulu.fi
}

\author{
Ville Niemelä \\ University of Oulu \\ Centre for Wireless Communications \\ Oulu, Finland \\ ville.niemela@oulu.fi
}

\begin{abstract}
This paper introduces remote activity monitoring scenario, which is deployed using indirect sensing of person's assisted living environment, i.e., providing monitoring without wearables. Wireless sensors are monitoring, e.g., temperature, $\mathrm{CO2}$, motion and electricity consumption. Based on the indirect sensing information, it can be noticed, e.g., that the monitored person has awakened in the morning since monitored devices has been used or some abnormal behavior is taking place in the assisted living environment. Sensor data is analyzed by dedicated algorithms to obtain information about user's behavior. Information collected by body sensors can be used to complement the information collected by the indirect sensing method. Remote sensing approaches are introduced in this paper at a general level and indirect sensing use case implementation in state-ofthe-art 5G Test Network in Oulu, Finland, is discussed in more details. Challenges and findings of this implementation work are discussed, and future improvement directions are proposed.
\end{abstract}

Keywords-Wireless healthcare, 5G Test Network (5GTN), wireless sensors, indirect monitoring, assisted living, IoT, care on demand

\section{INTRODUCTION}

Remote monitoring of patients is a well-known application scenario of wireless healthcare and assisted living. It has been proposed that sensors can be used, e.g., to monitor vital signs or fall detection of the patient [1] - [3]. Typically, these applications are targeted for monitoring chronically ill, disabled and elderly people at home, or in care facilities. Traditionally, remote monitoring systems are based on wireless body area network (WBAN) sensors, attached on the body, monitoring vital signs (heart rate, respiration rate, oxygen in blood, blood glucose level etc.) of the patient, or movements of the patient [1]. Indirect (contactless) sensing of physiological data has been somewhat less studied, but it has gained increased attention recently. Contactless sensing of physiological data can be divided into two categories: image-based and radar-based [1], in which data can be analyzed to make findings of the monitored person's behavior.

This paper focus on a third category: an indirect (i.e. passive) sensing approach by monitoring only the living environment of the target person(s). In this work, highly relevant experience and findings have been gained by implementing an end-to-end indirect monitoring system into a care facility located in Oulu, Finland. This remote monitoring is a "care" use case of the 5G Test Network (5GTN) [4], which is an innovative technological environment for testing solutions for future services and latest technological products. High-level architecture of the 5GTN is illustrated in Figure 1. 5GTN can be considered as a mobile operator since it has its own end-to-end network infrastructure with required frequency licenses. Currently it has four licenses for frequency bands relaying between 700 $\mathrm{MHz}$ and $3500 \mathrm{MHz}$, which provide radio coverage also for different low power wide area networks (LPWAN) designed technologies such as narrowband internet of things (NB-IoT) and category M1 (Cat M1). Additionally, radio access connectivity in 5GTN for different devices can be utilized through unlicensed technologies, e.g., Bluetooth low energy (BLE), Wi-Fi and low power, wide area networking (LoRaWAN). The evolved packet core (EPC) manages session states and authentications, routes data packets through the access networks and acts as the interface between LTE network and other packet networks. Through the EPC there is a connection anywhere in the internet and there are several virtual private network (VPN) connections generated to different remote locations when needed.

In this use case the wireless sensors are measuring temperature, $\mathrm{CO} 2$, motion and electricity consumptions to find out whether the patient's behavior is normal or abnormal, need of assist or care on demand based on longterm measurements, analysis and learning. Our approach works as an individual implementation, but the system can be complemented with additional WBAN and contactless image- and/or radar-based sensing information too. Combined WBAN sensor data and passive sensing data would allow more intelligent decisions based on data analysis, for example, WBAN sensors are able to detect abnormal physiological symptoms in real-time, where the passive monitoring is not capable for this. The benefit for the passive sensing is that it does not require anything from the person him- or herself. For example, the elderly people of the 2010s having assistance in their daily life were born and raised approximately in the 1920s and 1930s and may have reluctance for many technological solutions, especially wearables. 


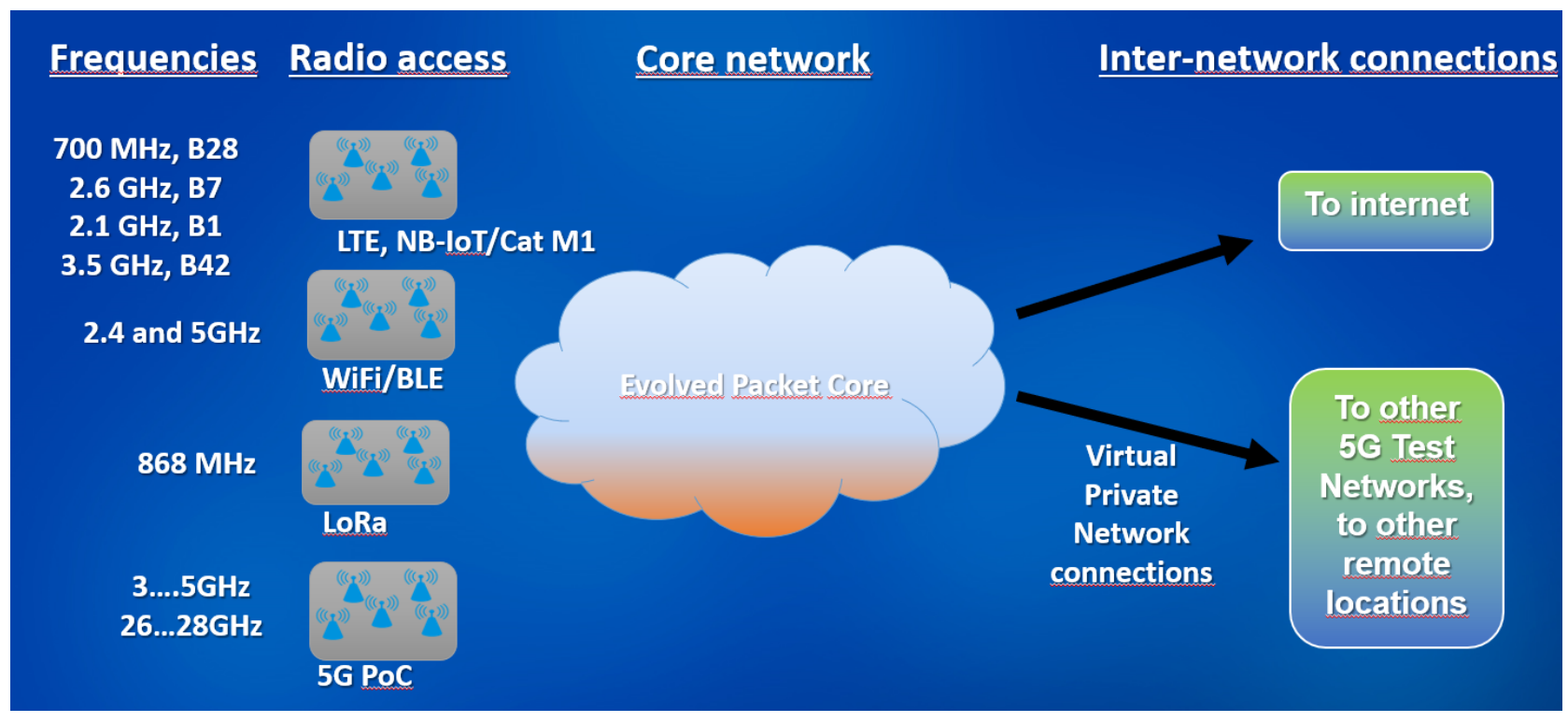

Figure 1. High-level architecture of 5G Test Network.

This paper introduces the indirect sensing approach, relevant wireless technologies and practical implementation and testing of the solution in a real-life assisted living care facility. Valuable findings are discussed based on the gained experience during this use case implementation life cycle.

\section{REMOTE MONITORING}

Digitalization and IoT is revolutionizing future healthcare services. Remote activity monitoring is well-known scenario that is proposed to enable savings for society and to improve patient's quality of life and safety by prolonging living at home even with illness or disabilities. At first, in this section is given an overview of different type of remote activity monitoring approaches proposed in the literature, dividing them to different sensing categories: contact-based, contactless and indirect sensing. Secondly, the most common technologies for wireless sensor nodes, which can be used in remote monitoring scenarios, are introduced.

\section{A. Sensing approaches}

There are various situations where remote patient monitoring is envisaged to be useful [1] - [3]. Previous works focus mostly on monitoring that is in contact with patient. In [1] authors found out that most of the monitoring systems are designed to following categories based on the diagnosed disease: heart and blood related diseases; fall detection and mobility related disease; brain, neurological system related diseases and mental health; and diabetes. Furthermore, the authors divided the sensing approaches to contact-based and contactless [1]. Their categorization is based on the traditional assumption that remote patient monitoring systems are designed to obtain physiological data from patients. In that case contact-based sensors are typical WBAN nodes and contactless sensors can measure physiological parameters from few meters distance from patient using non-invasive approach which has gained researchers attention in recent years [1]. In this work, we found that useful monitoring of a person can be also done using contactless sensing approach, which does not measure physiological parameters and is not targeted for monitoring any particular disease. Therefore, we propose a third category for sensing approaches, which is called indirect sensing; invisible and non-distruptive monitoring of a person's living environment. Below we introduce shortly different solutions based on these three categories to provide an insight to different approaches.

\section{1) Contact-based}

Physiological data are typically obtained by using sensors attached onto human body, i.e., they are in contact with human. The most common measures are electrocardiogram (ECG), electroencephalogram (EEG), heart rate, respiration rate, pulse oximetry, blood pressure, body temperature and blood glucose level. Many of these measurements can be done with a wireless measurement unit in a long-term based measurements, but for example, ECG and EEG are usually short term single measurement event with wired connections. Contact based measurements provide very accurate measurement information. With wireless devices, charging the device or battery replacement need to be taken into account and for elderly people specifically, there may exist some reluctance of using such devises. Surveys about contact-based remote monitoring systems are introduced e.g., in [1], [2], [5] and [6].

\section{2) Contactless}

Recent trend in patient sensing is to use contactless sensing as much as possible in remote health monitoring. Contactless sensing are mainly done by using image- and radar-based methods [1]. Image-based methods can be used, e.g., for heart-rate estimation, respiration rate and blood oxygen level measurements [7], [8]. Different vision-based monitoring systems (detection of illnesses, sleep monitoring and activity monitoring etc.) are surveyed e.g. in [9] and [10]. With the contactless monitoring, there are no requirements for person(s) to wear anything and generally, the equipment are power plugged.

\section{3) Indirect sensing}

In the future's smart homes, different type of sensors will be installed to monitor the environment, e.g. temperature, air 
quality, lighting and motion. Electricity and water consumptions are measured already but only for invoicing purposes and in general, not for real time. By combining different sensor information it is possible to make conclusions about the actions and condition of person(s) inside the home. For example, simple usage detection algorithm in both electricity and water consumption meters can reveal that in the morning an elderly person has waken up and that it is a proper time for assisting personnel to go for a routine morning visit. Another approach is to set up motion detectors to figure out whether an elderly people is awake in the morning or whether he or she stays awake or is wandering around the apartment during the night. In addition, it is possible to extend the purpose of different measurements to the condition of buildings and its devices.

In this work we categorize this kind of system as an indirect sensing approach for remote person monitoring which is also a non-invasive method and does not measure nor record patient's physiological data. Sensors for assisted living, such as passive infrared (PIR) sensors, motion sensors, vibration sensors, pressure sensors, power and water consumption sensors, cameras, and microphones can be used in passive sensing approach. Recent survey of assisted living sensors has been done in [11].

\section{B. Communication Technologies for Wireless Sensors}

There are various wireless technologies, which can be used to collect sensor data from the remote monitoring location, from the environment and monitored person body. A brief summary of wireless technologies that are commonly found in wireless sensing scenarios is presented here.

Bluetooth is a well-known wireless technology that has been around for over twenty years and has gone through several iterations, each improving or expanding the features of the previous one [5]. A low-power version, Bluetooth 4.0, also called Bluetooth Low Energy (BLE) was introduced in 2010. It consumes little power and can be operated with small power sources or by harvesting energy. It is widely used on a large variety of healthcare devices, e.g., heart rate and blood pressure monitors. It operates in the license-free $2.4 \mathrm{GHz}$ industrial, scientific and medical (ISM) band. Bluetooth 5.0 improves further this technology's suitability for the IoT applications by increasing range, speed and broadcasting capacity.

IEEE 802.15.6 [16] and ETSI SmartBAN [17] standards are the first ones tailored specifically for WBANs, which can be used in health, medical, and sport applications. The IEEE Std. 802.15.6 offers three physical (PHY) layer options: narrowband (NB), ultra-wideband (UWB) and human body communications. The medium access control (MAC) layer for this standard has three different modes and thus it offers flexibility for operation in different scenarios. The ETSI SmartBAN standard is more recent than IEEE Std. 802.15.6. The frequency of operation of this standard is between 2.401 $\mathrm{GHz}$ and $2.481 \mathrm{MHz}$ with $2 \mathrm{MHz}$ spacing, i.e., similar to the classic Bluetooth. This protocol uses separate channels for data and control messages and like the IEEE Std. 802.15.6 it has different channel access modes [17].

ANT/ANT+ [18] is low-power proprietary protocol designed for low data rate sensor networks that can be configured in different topologies, e.g. peer-to-peer, star, and mesh. It operates in the $2.400-2.524 \mathrm{GHz}$ band, the ANT+ devices operates in the $2.457 \mathrm{GHz}$ frequency. The ANT channels are $1 \mathrm{MHz}$ wide. The protocol is time division multiple access based with channel hopping capabilities. The flexibility features of this protocol allow ANT devices to adapt to hostile conditions by changing the amount of control overhead depending on the amount of experienced interference.

The IEEE 802.11 [19] is an ever-evolving set of PHY and MAC layer standards specifications for the implementation of wireless local area network (WLAN) communications. The original version of this standard was released in 1997. Ever since then it has been revised increasing the range of its features, in particular the bit rates. It has version operating in the $2.4 \mathrm{GHz}$ frequency bands $(802.11 \mathrm{~b} / \mathrm{g} / \mathrm{n})$, as well in the $5 \mathrm{GHz}$ region $(802.11 \mathrm{a} / \mathrm{ac} / \mathrm{n})$.

ZigBee [20] is the most well-known technology for wireless sensor networks (WSNs). ZigBee defines upper layers on top of the IEEE Std. 802.15.4 PHY and MAC [21] and most of the solutions are operating at congested $2.4 \mathrm{GHz}$ band.

Long range (LoRa) technology is operating at sub-GHz bands to enable long-range IoT connectivity. LoRaWAN defines the communication protocol and system architecture for the network while the LoRa physical layer enables the long-range communication link [22]. LoRaWAN specification is intended for wireless battery-operated things in regional, national or global network. LoRaWAN targets to fulfill key requirements of IoT applications such as secure bidirectional communication, mobility and localization services. Communication between end-devices and gateways is spread out on different frequency channels and data rates. The selection of the data rate is a trade-off between communication range and message duration. Due to the spread spectrum technology, communications with different data rates do not interfere with each other and create a set of "virtual" channels increasing the capacity of the gateway. LoRaWAN data rates range from $0.3 \mathrm{kbps}$ to $50 \mathrm{kbps}$. Anoth-er similar type sub-GHz solution for low-power widearea networks is provided by Sigfox [23].

NB-IoT, is a recent narrowband radio technology developed for the IoT scenarios by the 3rd Generation Partnership Project (3GPP) [24]. Indeed, 3GPP has made a major effort to address the IoT market by actively developing solutions, such as NB-IoT, enhanced machine-type communication (eMTC or LTE-M) and extended coverage GSM (EC-GSM) [25], which will operate in licensed cellular bands, in contrast to all the other solutions presented here. NB-IoT and eMTC bring many enhancements and optimizations to LTE that will reduce IoT device complexity, enable multi-year battery life, and provide deeper coverage to reach challenging locations such as deep inside buildings. The new technologies can leverage existing LTE infrastructure and spectrum, enabling easier coexistence control in comparison to unlicensed band. Therefore, these technologies may become popular also in healthcare and medical applications.

\section{CARE USE CASE IMPLEMENTATION IN 5GTN}

$5 \mathrm{G}$ Test Network (5GTN) is managed and developed by the University of Oulu and Technical Research Centre of Finland (VTT) in collaboration with over 20 industry partners [4]. It is a scalable research and development 


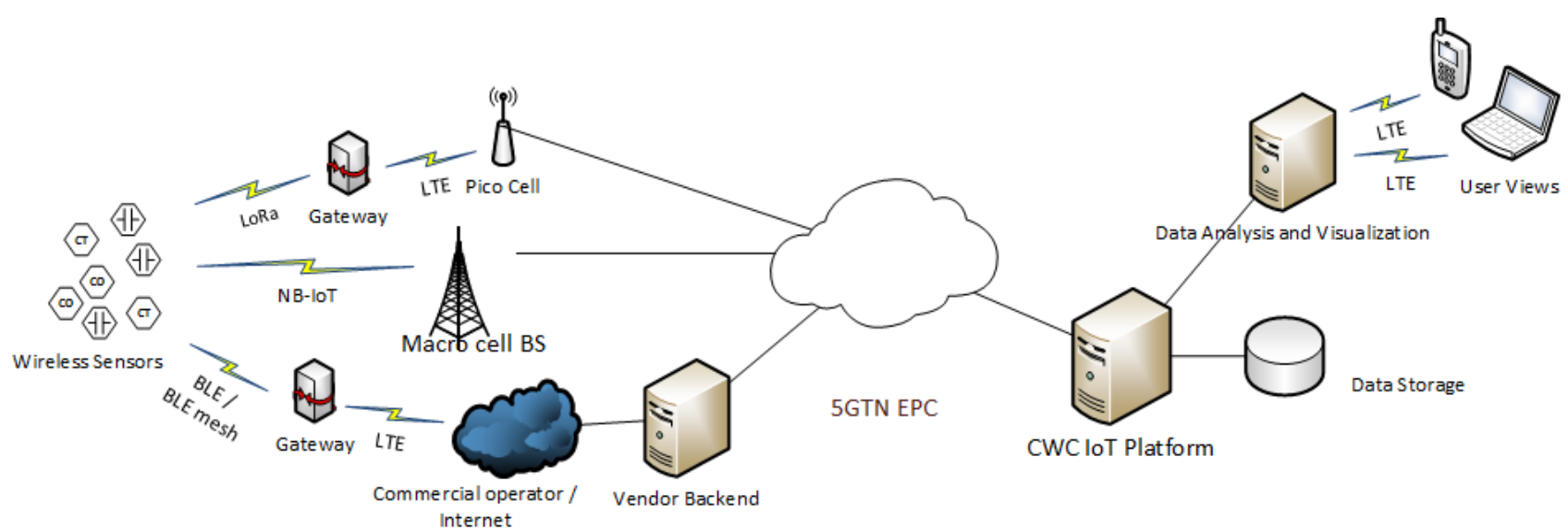

Figure 2. Architecture of the indirect sensing approach implementation utilizing the 5GTN.

environment for experimenting $5 \mathrm{G}$ technology, future business models and services. 5GTN has been deployed in many sites and it already supports multiple use cases, such as care, media, sport and virtual reality [26]. In this section, the care use case is discussed in more details. The care use case studies $5 \mathrm{G}$ and IoT technologies utilization for smart home and quality of service improvement in home for elderly people. Implemented scenario can be generalized to indirect monitoring of patients, e.g., memory ill people at home or other type of care facilities. In this particular use case, the target group is elderly people needing assistance for daily routines but each of them living in their own apartments. Sensing information will enable service providers to apply ideal timing of daily care. For example, patient's wake-up time may vary and assistance should be adapted to the morning rhythm of each individual resident to enable efficient resource usage and comfortable, ideal timed service and assistance - care on demand.

\section{A. Network Architecture}

The high-level network architecture of the care use case is illustrated in Figure 2. The home for elderly is a multifloor apartment and can be considered as a remote site, consisting of two pico cell base-stations (on separate floors), which are connected to the 5GTN EPC via VPN connection. Pico cells provide indoor coverage throughout the building and they are able to collect information from sensor nodes that are installed to the patient's rooms. There is also a macro cell providing the coverage for NB-IoT capable sensors. Connectivity to wireless sensors are implemented using NBIoT and LoRaWAN technologies as illustrated in Figure 2

There are seven different measured parameters that are being measured and the information is transmitted wirelessly to a data base. The measured parameters are power consumption, temperature, humidity, luminance, oxygen level and motion detector.

Sensor data is delivered to IoT system and data analysis servers, which provide appropriate data for the end-user application. The goal of this architecture implementation is to study different radio access types and protocols, sensors from several vendors, and integration of IoT platforms in a real environment. Data analysis, algorithms and data visualization is an essential part of a real end-user service, e.g., for personnel and later for residents and their relatives.

\section{B. Sensors}

The care use case was implemented using only sensors, which do not require intervention of users and are comfortable for the patients since they are non-invasive and contactless. In this case, the IoT sensors detect motion inhome, measure power consumption and luminance, $\mathrm{CO} 2$, humidity and temperature. One of the key requirements is privacy - the home is home, cameras and microphones were excluded on purpose. Additionally, bathroom was left out of sensors also for privacy reasons. Sensible solutions based on audio and video could be included in the future architectures as well as the monitoring the whole apartment.

\section{Data storage and analysis}

The data collected by sensors and personnel are stored in the databases. In the future, it is planned that the sensor data is analyzed by dedicated algorithms, and the results are available for personnel in real time. In this study we present the concept and focus on preliminary analysis. Based on the future analysis, care on demand i.e. optimal, ideally timed service can be provided for the residents. The data and results are very sensitive, so security, privacy and data protection are taken and for any future use cases, should be taken into consideration in all levels with high priority.

\section{RESULTS AND DISCUSSION}

During the service design it was identified that the most valuable data are motion detection, water and electricity consumption sensors' information. Actions like the usage of microwave, coffee machine, water tabs, washing machine and shower, each have a unique consumption fingerprint. The sensor data could provide insights to a person behavior in-home. Long term monitoring might enable proactive health care, detection of slowly changing behavioral and health changes. 


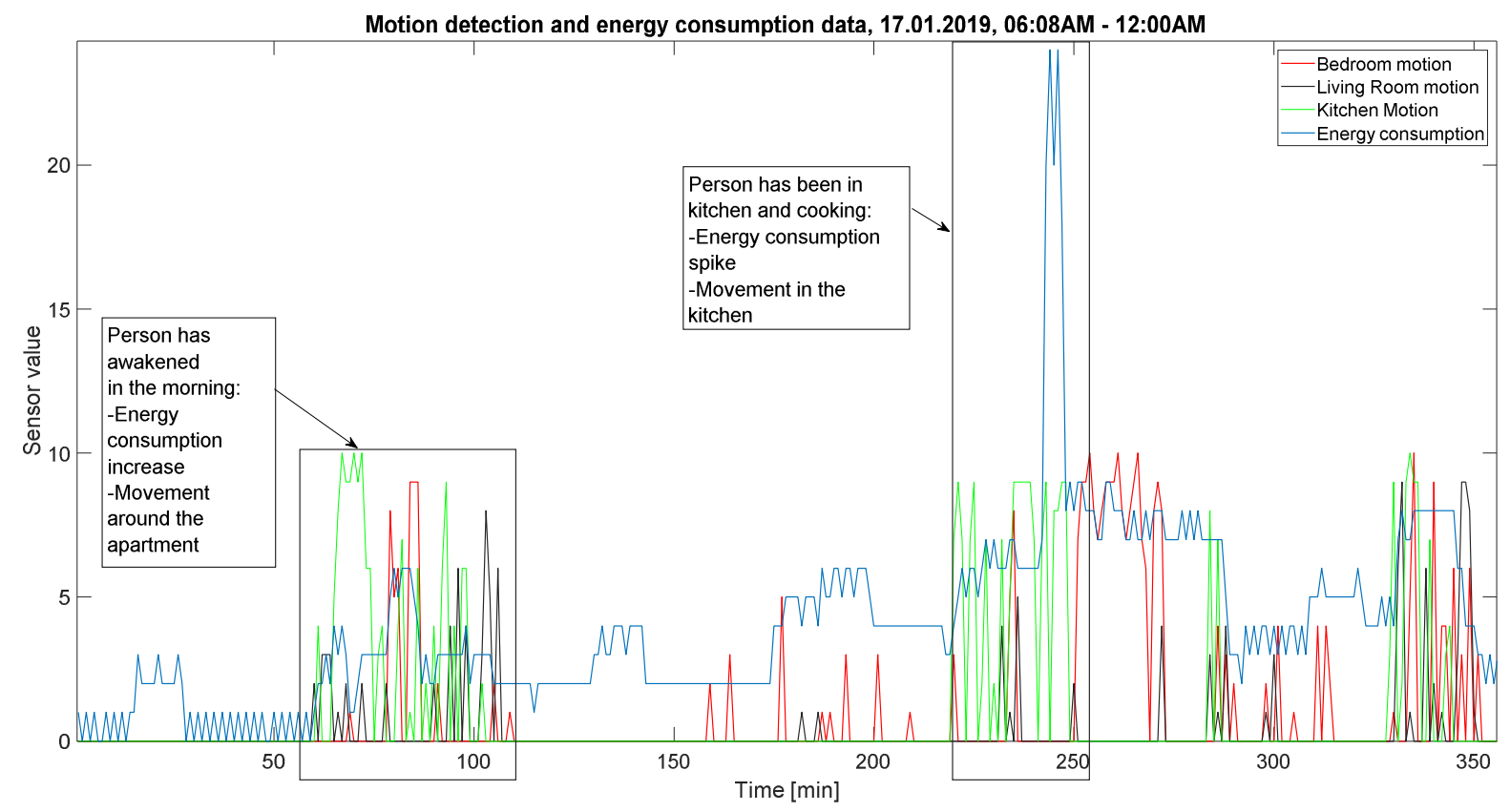

Figure 3. Energy consumption and motion detection data for a one day between 6.08 AM and noon.

Figure 3 shows an example results of the analysis of energy consumption and motion detection sensors' data from the monitored apartment collected during a one day morning until the noon. It can be observed that based on this data it can be made conclusions about the patients' daily routines. In the morning after 7 AM energy consumption has increased most probably since the lights have been turned on. Also movement around the apartment has occured then. Around 10 AM monitored person has stayed in the kitchen and there is a spike in energy consumption, most probably due to cooking food or coffee. Based on this result it can be stated that in the patients' behaviour analysis, energy consumption and motion detection are important features.

Based on the long term monitoring data it is for example possible to plan the schedule of assisting personnel using the data averaging. An example results about data analysis based on data averaging is shown in Figure 4.
Potential extensions of the care use case include additional views of results tailored for residents themselves, their relatives, researchers and doctors of elderly people and other groups needed assisted living. In the future, additional sensors can also be included to the system, e.g., physiological data measurements using WBANs would provide valuable information to be used in data analysis and intelligent decisions. In some cases audio, video or even smells, i.e. microphones, cameras and a sort of artificial nose could be used too. However, it must be kept in mind that privacy requirement will be the most strict in these cases. It will be also more difficult to achieve user acceptance for monitoring private and very sensitive data. In any case, it is expected that in future this is the development direction and the proposed architecture enables straightforward collection and analysis of any sensor data.






\section{CONLUSIONS}

This article discussed remote and indirect monitoring scenarios for wireless healthcare and assisted living systems. An example implementation of remote activity monitoring scenario, utilizing state-of-the-art 5G Test Network in Finland, was introduced. Implementation includes a complete end-to-end architecture, which can be used to provide valuable end-user services based on wireless sensing and data analysis. Same approach can be used for remote monitoring of patients in home and in care facilities. This work included only indirect sensing, which can be used to monitor user behavior by collecting information from patient's home. Example results of the sensor data analysis showed that the indirect sensing can be used to find out useful information about the behaviour of monitored person. Deployed technical architecture enables that additional sensors, such as WBAN sensors can easily be added to the system in future. Future work includes also more detailed performance evaluation of the technical solution and more comprehensive analysis of the data and its benefits in practical applications.

\section{ACKNOWLEDGMENT}

This research has been financially supported by EU regional funds through $5 \mathrm{GTN}+$ project, and by Academy of Finland 6Genesis Flagship (grant 318927). Authors want to express compliments to Technical Research Centre of Finland (VTT) and Indalgo about fruitful collaboration during this work.

\section{REFERENCES}

[1] Malasinghe, L. P., Ramzan N. and Dahal K.: Remote patient monitoring: a comprehensive study. Journal of Ambient Intelligence and Humanized Computing, pp. 1-20. Springer, Berlin Heidelberg (2017).

[2] Chen $M$ et al.: A survey of recent developments in home M2M networks. IEEE Commun Surv Tutor 16(1):98-114, 2014

[3] S. Movassaghi, M. Abolhasan, J. Lipman, D. Smith and A. Jamalipour. :Wireless Body Area Networks: A Survey, in IEEE Communications Surveys \& Tutorials, vol. 16, no. 3, pp. 1658-1686, Third Quarter 2014.

[4] 5G Test Network (5GTN), https://5gtn.fi/

[5] Silva BMC et al.: Mobile-health: a review of current state in 2015. J Biomed Inf 56:265-272, 2015.

[6] Sawand A, Djahel S, Zhang Z, Naït-Abdesselam F.: Toward energyefficient and trustworthy eHealth monitoring system. China Commun 12(1):46-65, 2015.
[7] Hassan MA, Malik AS, Fofi D, Saad N, Karasfi B, Ali YS, Meriaudeau F (2017) Heart rate estimation using facial video: a review. Biomed Signal Process Control 38(Supplement C).

[8] McDuff DJ, Estepp JR, Piasecki AM, Blackford EB.: A survey of remote optical photoplethysmographic imaging methods. In: 2015 37 th annual international conference of the IEEE engineering in medicine and biology society (EMBC), pp 6398-6404, 2015.

[9] Sathyanarayana S, Satzoda RK, Sathyanarayana S, Thambipillai S.: Vision-based patient monitoring: a comprehensive review of algorithms and technologies. J Ambient Intell Hum Comput., 2015.

[10] Baig MM, Gholamhosseini H.: Smart health monitoring systems: an overview of design and modeling. J Med Syst 37(2):9898, 2013.

[11] F. Erden, S. Velipasalar, A. Z. Alkar and A. E. Cetin, "Sensors in Assisted Living: A survey of signal and image processing methods," in IEEE Signal Processing Magazine, vol. 33, no. 2, pp. 36-44, March 2016.

[12] Crosby Garth V. Tirthankar Ghosh Renita Murimi and Craig A. Chin.: Wireless Body Area Networks for Healthcare: A Survey. International Journal of Ad Hoc Sensor \& Ubiquitous Computing Vol. 3 no. 3, June 2012.

[13] Kim B-S, Kim KH, Kim K-I. A Survey on Mobility Support in Wireless Body Area Networks. Fortino G, Ghasemzadeh H, Li W, Zhang Y, Benini L, eds. Sensors (Basel, Switzerland). 2017;17(4):797.

[14] Q. Zhang and M. Karunanithi, "Feasibility of unobstrusive ambient sensors for fall detections in home environment," 2016 38th Annual International Conference of the IEEE Engineering in Medicine and Biology Society (EMBC), Orlando, FL, 2016, pp. 566-569.

[15] Bluetooth SIG, https://www.bluetooth.com/

[16] IEEE Std. 802.15.6: IEEE Standard for Local and metropolitan area networks - Part 15.6:Wireless Body Area Networks. Standard, The Institute of Electrical and Electronics Engineers, Inc., 2012.

[17] M. Hämäläinen et al., "ETSI TC SmartBAN: Overview of the wireless body area network standard," International Symposium on Medical Information and Communication Technology (ISMICT), 2015.

[18] ANT/ANT+, https://www.thisisant.com/

[19] IEEE 802.11 LAN Working Group, http://www.ieee802.org/11/

[20] ZigBee Alliance, http://www.zigbee.org/

[21] IEEE Standard for Low-Rate Wireless Networks,"IEEE Std 802.15.42015 (Revision of IEEE Std 802.15.4-2011)” April 22, 2016.

[22] LoRa alliance, "A technical overview of LoRa and LoRaWAN" white paper, https://www.loraalliance.org/portals/0/documents/whitepapers/LoRaWAN101.pdf, November 2015.

[23] Sigfox, https://www.sigfox.com/en.

[24] 3GPP new release, http://www.3gpp.org/news-events/3gppnews/1785-nb_iot_complete

[25] Nokia white paper, "LTE evolution for IoT connectivity," 2017.

[26] Kanstrén, T. et al.: Vertical Use Cases in the Finnish 5G Test Network, European Conference on Networks and Communications (EuCNC), June 2018. 